\title{
Low-Frequency Stimulation Depresses the Primate Anterior- Cingulate-Cortex and Prevents Spontaneous Recovery of Aversive Memories
}

\author{
Oded Klavir, Rotem Genud-Gabai, and Rony Paz \\ Department of Neurobiology, Weizmann Institute of Science, Rehovot, Israel 76100
}

Functional abnormalities in the dorsal-anterior-cingulate-cortex (dACC) underlie anxiety disorders and specifically post-traumatic stress disorder (PTSD). Promising and common behavioral approaches have limited effectiveness and many subjects exhibit spontaneous recovery of fear, as also evident in animal models following extinction training. Here, we use low-frequency stimulation (LFS), a protocol shown to induce long-term depression, with the aim of affecting synaptic plasticity induced by fear acquisition and extinction. We use aversive conditioning of either tone or visual stimuli paired with an aversive air-puff to the eye in a trace-conditioning paradigm. We find that LFS in the nonhuman primate (Macaca fascicularis) dACC, when combined with extinction training, was successful in preventing spontaneous recovery of the memory $24-72 \mathrm{~h}$ following extinction. We simultaneously record single-units and local-fieldpotentials across the dACC, and show that LFS gradually depressed evoked responses. Moreover, this decrease in neural excitability predicted the successful reduction of overnight spontaneous recovery on a day-by-day basis. Finally, we show that this effect occurs when using either visual or auditory modality as the conditioned stimulus, and that the reduction was specific to the conditioned modality. Our results suggest that the primate dACC is actively involved in maintaining the original aversive memory, and propose that a combination of LFS with behavioral therapy might significantly improve treatment in severe cases.

\section{Introduction}

The neural mechanisms underlying acquisition and extinction of fear-associations have been the focus of growing interest and are used as an effective model for anxiety disorders (Delgado et al., 2006; Milad et al., 2006; Yehuda and LeDoux, 2007). Extinction training is being used as a core behavioral treatment for trauma and anxiety disorders, but with limited effectiveness (Foa, 2006; Myers and Davis, 2007; Yehuda and LeDoux, 2007; Quirk et al., 2010). This is possibly because extinction training does not directly modify the existing fear-memory but instead leads to the formation of a new memory that inhibits activation of the initial trace. Therefore, after enough time has passed, previously extinguished fear can re-emerge in the form of spontaneous recovery (Pavlov, 1927; Robbins, 1990; Bouton, 2006; Schiller et al., 2008; Monfils et al., 2009). Although different pharmacological and behavioral approaches are used (Yehuda and LeDoux, 2007), it is still largely unknown whether and how to use safe invasive methods to improve the effectiveness of behavioral treatment.

Received Dec. 28, 2011; revised March 7, 2012; accepted March 29, 2012.

Author contributions: 0.K., R.G.-G., and R.P. designed research; 0.K., R.G.-G., and R.P. performed research; 0.K., R.G.-G., and R.P. analyzed data; $0 . K$. and R.P. wrote the paper.

This work was supported by an Israel Science Foundation grant (\#430/08) and European Research Council Reintegration and Starting Grants to R.P. We thank Yossi Shohat for invaluable contribution for the animals work and welfare, Dr. Eilat Kahana and Dr. Gil Hecht for help with medical and surgical procedures, and Dr. Edna Furman-Haran and Nachum Stern for MRI procedures.

Correspondence should be addressed to Dr. Rony Paz, Department of Neurobiology, Weizmann Institute of Science, Rehovot, Israel 76100.E-mail: rony.paz@weizmann.ac.il.

DOI:10.1523/JNEUROSCI.6481-11.2012

Copyright $\odot 2012$ the authors $\quad 0270-6474 / 12 / 328589-09 \$ 15.00 / 0$
A commonly accepted model developed in rodents suggests that the amygdala mediates fear acquisition and expression (LeDoux, 2000; Maren and Quirk, 2004; Pape and Pare, 2010) and is modulated by inputs from two cortical regions: the prelimbic cortex (PL) which mediates and enhances fear expression, and the infra-limbic cortex (IL) which mediates fear extinction (Sierra-Mercado et al., 2011). In primates, the dorsal-anteriorcingulate-cortex (dACC) has similar anatomy and connectivity to that of the rodent PL (McDonald, 1998; Stefanacci and Amaral, 2002; Ghashghaei et al., 2007), and a similar functional role is supported by human studies showing its involvement in fear expression (Dunsmoor et al., 2007; Milad et al., 2007) and abnormal functionality in anxiety disorders (Liberzon et al., 1999; Bremner et al., 2005; Straube et al., 2006; Milad et al., 2009; Pannu Hayes et al., 2009; Shin et al., 2011).

Both acquisition and extinction of fear learning are thought to be dynamically mediated by synaptic plasticity in amygdalaprefrontal circuits (Goosens and Maren, 2004; Likhtik et al., 2005; Herry et al., 2008; Amano et al., 2010; Duvarci et al., 2011). Artificially, synaptic plasticity and strength-modulations can be induced by different protocols of electrical microstimulation, and specifically, low-frequency stimulation (LFS) was shown to reduce responsiveness and induce similar effects to long-termdepression (Wang and Gean, 1999; Maroun, 2006; Kim et al., 2007). We therefore hypothesized that LFS in the primate dACC could reduce neuronal excitability and responsiveness in this region, and when combined with extinction training, result in decreased spontaneous recovery of the original aversive memory. To test this, we trained monkeys on an aversive (airpuff to the 
a

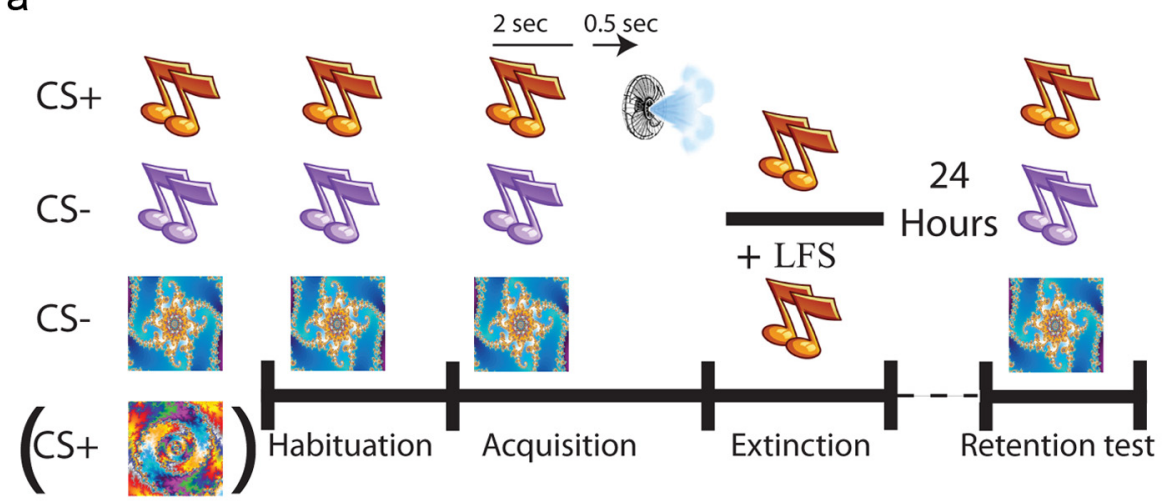

C
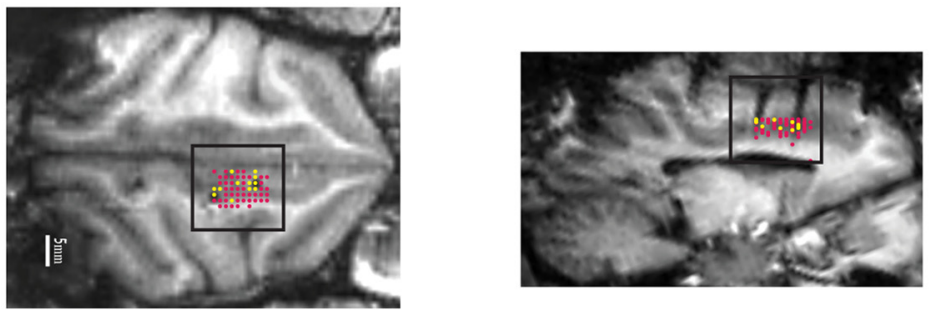

d

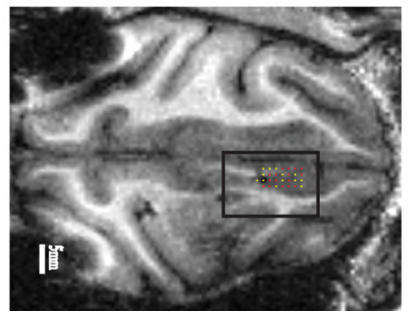

b
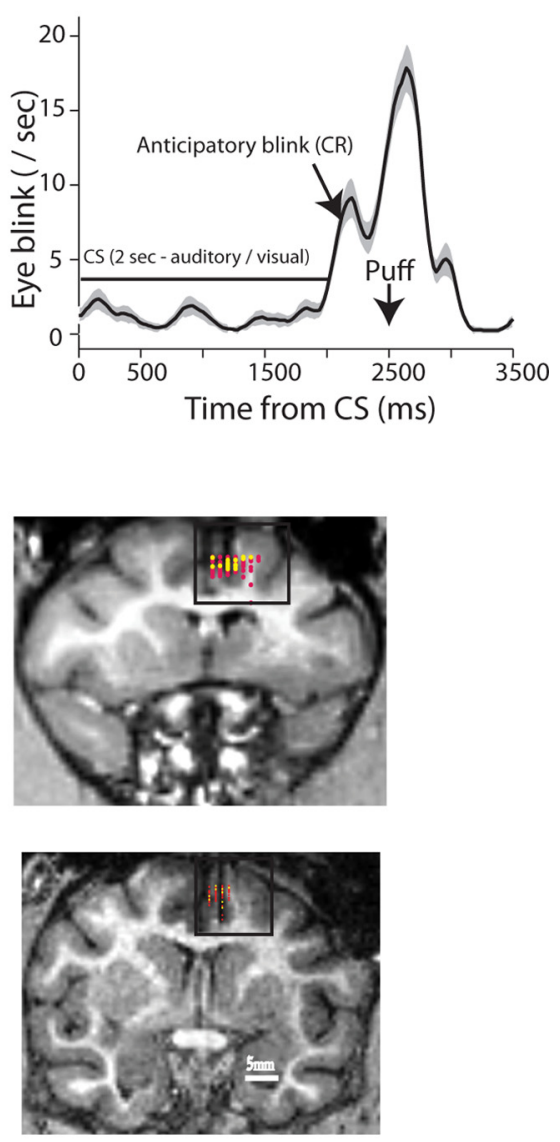

Figure 1. Behavioral paradigm, stimulation and recording locations. $\boldsymbol{a}$, Each session included three new stimuli, one CS +, and two CS - , one of which was the same modality of the CS +, and one was the other modality (stimuli were pure-tones or fractal images, all easily distinguishable perceptually and discriminated during learning). A new session started with a habituation phase, followed by an acquisition phase, and an extinction phase. All sessions were preceded by a retention test where we presented the three CS of the previous day and tested for spontaneous recovery of memory. In behavior-only sessions, there was only behavioral extinction, and in stimulation sessions the extinction included LFS in the dACC of $1 \mathrm{~Hz}$ for 15 min. $\boldsymbol{b}$, The behavioral learned conditioned response (CR), averaged over all sessions in the trace-conditioning paradigm used. The $\mathrm{CS}$ was presented for $2 \mathrm{~s}$, then after $500 \mathrm{~ms}$ break came the air-puff. The animals learned and started eye-blink/closure immediately after the end of the $C S$ as an anticipatory response. $\boldsymbol{c}, \boldsymbol{d}, \mathrm{MRl}$ images of anatomical sections for the two animals with electrode tracks (black traces and dots), with overlaid recording locations (yellow dots) and stimulation locations (red dots) aligned by the position of the electrodes. MRIs were performed before, during, and after the recording period.

eye) conditioning task. The acquisition was followed by an extinction session (presentation of the CS alone). Some sessions had standard extinction training (behavior-only), and in some randomly intermingled sessions, the same extinction training was combined with LFS in the dACC. We simultaneously recorded field-potentials and single-unit activity from electrodes throughout the dACC. We then tested for retention of the aversive memory in the following day ( $24 \mathrm{~h}$ overnight retention), and compared the effect of LFS during extinction to that of behavior-only.

\section{Materials and Methods}

Animals. Two male Macaca fascicularis (4-7 kg) were implanted with a recording chamber $(27 \times 27 \mathrm{~mm})$ above the right anterior cingulate cortex (ACC, broadman 24/32), under deep anesthesia and aseptic conditions. All surgical and experimental procedures were approved and conducted in accordance with the regulations of the Weizmann Institute Animal Care and Use Committee (IACUC), following NIH regulations and with Association for Assessment and Accreditation of Laboratory Animal Care accreditation. Food, water, and enrichments (e.g., fruits and play instruments) were available ad libitum during the whole period, except before medical procedures.
Behavioral paradigm and recording locations. During each session the monkeys engaged in a $500 \mathrm{~ms}$ trace classical conditioning task with a random intertrial interval (ITI) of $25 \mathrm{~s}$ on average (Fig. 1a). Conditioned stimulus $(\mathrm{CS}+$ ) of either a new pure tone or a new fractal cue (Chaos Pro 4.0 program; www.chaospro.de) was paired with an unconditioned aversive stimulus (US) of an air puff (150 ms duration; 3-5 bars; located proximally $5 \mathrm{~cm}$ from the left eye). Two other stimuli were presented without the aversive air puff outcome (CS-), a different pure tone and a different fractal cue. Hence, in each session, there was an auditory or a visual CS +, and one CS - of the same modality and one CS - of the other modality. Each new session started with a "habituation" stage where the three CSs were presented randomly ( 6 trials of each); followed by the "acquisition" stage described above $(15+15+15$ trials); and an "extinction" stage, where the CS+ was presented again without US (15 trials). Before the beginning of each session, we first tested for overnight retention of associations by presenting the three CSs from the previous day $(7+7+7$ trials $)$.

There were 92 sessions overall, 65 without stimulation (behavior only), and 27 with stimulation (behavior + LFS). Of the 65, the CS+ was auditory in 28 , and visual in 37 . Of the 27 , the CS + was auditory in 15 , and visual in 12 . Behavior-only and stimulation sessions were randomly intermingled during the recording period. We also verified that there is 
no effect of early sessions (the first half of the recording period) versus late sessions (the second half, two-way ANOVA).

MRI-based electrode positioning. Anatomical MRI scans were acquired before, during, and after the recording period. Images were acquired on a 3-tesla MRI scanner: (MAGNETOM Trio, Siemens) with a CP knee coil (Siemens). T1 weighted and 3D gradient-echo (MPRAGE) pulse sequence was acquired with TR of $2500 \mathrm{~ms}$, TI of $1100 \mathrm{~ms}$, TE of $3.36 \mathrm{~ms}, 8^{\circ}$ flip angle, and two averages. Images were acquired in the sagittal plane, $192 \times 192$ matrix and $0.8^{3} \mathrm{~mm}$ or $0.6^{3} \mathrm{~mm}$ resolution. A first scan was performed before surgery and used to align and refine anatomical maps for each individual animal (relative location of the ACC and anatomical markers such as the interaural line and the anterior commissure; confirmed using Martin and Bowden (2000) and Saleem and Logothetis (2007). We used this scan to guide the positioning of the chamber on the skull at the surgery. After surgery we performed another scan with two electrodes directed toward the ACC, and 2-3 observers separately inspected the images and calculated the ACC anterior-posterior and lateral-medial borders relative to the electrodes. The depth of the ACC was calculated from the dura surface.

Recordings. The monkeys were seated in a dark room and each day, three microelectrodes (0.6-1.2 $\mathrm{M} \Omega$ glass/narylene-coated tungsten, Alpha Omega or we-sense) were lowered inside a metal guide (Gauge 25xxtw, OD:0.51 mm, ID:0.41 mm, Cadence) into the brain using a head-tower and electrode-positioning system (Alpha-Omega). The guide was lowered to penetrate and cross the dura and stopped once in the cortex. The electrodes were then moved independently further into the ACC (we performed 4-7 mapping sessions in each animal by moving slowly and identifying electro-physiological markers of firing properties tracking the known anatomical pathway into the ACC). Electrode signals were preamplified, $0.3 \mathrm{~Hz}-6 \mathrm{KHz}$ bandpass filtered and sampled at $25 \mathrm{Khz}$; and on-line spike sorting was performed using a template-based algorithm (Alpha Lab Pro, Alpha Omega). We allowed $30 \mathrm{~min}$ for the tissue and signal to stabilize before starting acquisition and behavioral protocol. At the end of the recording period, off-line spike sorting was further performed for all sessions to improve unit isolation (offline sorter, Plexon).

Stimulation. Stimulation was performed through the recording microelectrodes using a stimulus generator (STG 4004, Multi Channel System, MCS) The stimulation was monopolar, and the reference was the general ground (established by connecting all cranial metal connections), which was common to all electrodes. Stimulation was biphasic rectangular pulse of $200 \mu \mathrm{s}-90 \mu \mathrm{A}$ followed immediately by $200 \mu \mathrm{s} 90 \mu \mathrm{A}$. A constant interval of $999.6 \mathrm{~ms}$ was kept between the end of one stimulus and the initiation of the next one, leading to a $1 \mathrm{~Hz}$ low-frequency stimulation. During each stimulation session, all three electrodes were used for recording until stimulation phase was due, in which time recorder was detached and stimulator was attached to one electrode, which became the stimulating electrode.

Behavior. A computerized digital video camera for night conditions (Provision-Isr) recorded the monkey's left eye at $50 \mathrm{~Hz}$. Video analysis was performed on custom-made software implemented on Matlab to identify periods when the animal closed the eye. We validated the algorithm by random samples from several recording days and found it to be consistent with the judgments of a human observer for $>95 \%$ of the reported eye-blinks.

Statistical analysis. All results concerning behavior were analyzed using a repeated-measures ANOVA with eye-blink width as dependent variable, Significant interactions were followed by post hoc least significant difference comparisons, and for all comparisons, significance was assumed at $p<0.05$. Extinction analysis included main factors of trial ( 15 trials repeated measure) and stimulation (stimulation days/nonstimulation days). As there was no difference in performance between days in which retention was conducted 72 and $24 \mathrm{~h}$ after extinction, all different days were combined. Retention analysis included main factors of trial ( 7 trials repeated measure) and stimulation (stimulation days/nonstimulation days) referring to the day of the corresponding extinction. Spontaneous recovery analysis included main factors of learning phase (two repeated measure of two last extinction trials average and first trial of retention) and stimulation (stimulation days/nonstimulation days) re- ferring to the day of the corresponding extinction. Spontaneous recovery of the two different sensory modalities analysis included main factors of learning phase (two repeated measure of two last extinction trials average and first trial of retention, identical results were obtained when using either 1 or 3 last trials of extinction), modality (auditory CS plus/visual CS plus) and stimulation (stimulation days/nonstimulation days) referring to the day of the corresponding extinction.

\section{Results}

We tested the effect of LFS in the dACC of two monkeys. In each session, the animal was first habituated to two new pure-tones and one new visual fractal (or two fractals and one pure-tone, Fig. 1a). Two of these were then assigned as CS-, and one was assigned as the CS+ and paired with an air-puff to the eye in a trace-conditioning paradigm (Fig. $1 b$ ), shown to activate neurons in the primate amygdala in a similar task (Paton et al., 2006). Our design of one CS - of the same modality and one of the other modality allowed us to test for specificity of the memory. Ten minutes following acquisition, the animals underwent an extinction paradigm where the $\mathrm{CS}+$ was presented alone. Immediate extinction allows testing of complete extinction in the same session, yet strengthens overnight retention (spontaneous recovery) of the original association (Maren and Chang, 2006; Schiller et al., 2008; Woods and Bouton, 2008). In stimulation sessions/d ( $n=$ 27 sessions), extinction training was accompanied by mild nonspecific LFS ( $1 \mathrm{~Hz}, 0.4 \mathrm{~ms}$ pulse width, $90 \mu \mathrm{A}$ amplitude) in the dACC during the whole extinction period ( $15 \mathrm{~min}, 900$ pulses overall), and in nonstimulation sessions it was not ( $n=65$ sessions, "behavior-only"). Stimulation and behavior-only sessions were randomly intermingled throughout the recording period. Each session started with a retention test for the three CSs of the previous day. We simultaneously recorded single-unit activity and local-field-potentials (LFP) in the dACC. Location of stimulation and recording electrodes were confirmed by alignment to multiple MRI sessions performed with electrodes (Fig. $1 c, d$ ).

\section{Low-frequency stimulation prevents spontaneous recovery}

Aversive learning and memory in the different phases was measured by the overall width of anticipatory eye closure (eyeblinks), which significantly increased after the CS but before the US (Fig. $1 b, p=0.001, t=3.27$, paired $t$ test of first and last trial in acquisition), and was differential at the end of acquisition across the $\mathrm{CS}+$, the $\mathrm{CS}-$, and the CS - of the other modality ( $p=0.01, F=4.65$, ANOVA, $p<0.02$ comparing $\mathrm{CS}+$ to either $\mathrm{CS}-$, and $p>0.1$ comparing the two CS - , post hoc tests). Extinction training reduced behavior significantly until plateau (Fig. $2 a, F_{(14,980)}=2.748, p<0.001$, main effect of trials; Fisher LSD post hoc test of trial main effect revealed the first two trials were significantly different from the last four trials, $p<0.001$ in all post hoc comparisons), and this was not significantly influenced by LFS (repeated measures 2-way ANOVA of stimulation* trials: $F_{(14,980)}=0.254 p=0.997$ and main effect of stimulation $\left.F_{(1,70)}=3.2442 p=0.076\right)$. There was no difference in acquisition between the groups $\left(t_{(90)}=0.087, p=0.452\right.$, Fig. $2 a$, leftmost points).

However, memories that were extinguished with LFS showed little or no spontaneous recovery when tested $24 \mathrm{~h}$ later, especially when compared with memories extinguished without LFS (Fig. $2 a, F_{(1,82)}=10.613, p<0.005$, main effect of stimulation). This effect is validated by comparing directly the end-of-extinction versus beginning of retention [Fig. $2 b, F_{(1,88)}=4.8564, p<0.05$, interaction of stimulation ${ }^{\star}$ learning (extinction/retention); with a Fisher LSD post hoc test showing a significant increase in eye-blink 
length in retention beginning compared with extinction end (spontaneous recovery) only in nonstimulation days $(p<0.0001)$, while no significant effect was witnessed in stimulation days $(p>0.5)]$, and was similar in the two animals (no interaction of monkey with either stimulation: $F_{(1,86)}=$ $0.150, p>0.5$, learning $F_{(1,86)}=0.112$, $p>0.5$ or monkey ${ }^{\star}$ stimulation ${ }^{\star}$ learning $\left.F_{(1,86)}=0.005, p>0.5\right)$. Moreover, the same qualitative result was obtained when testing the memory after $72 \mathrm{~h}$ (there were several over-the-weekend sessions, $n=4$ for LFS, $n=15$ for behavior-only, Fig. 2a).

We stimulated in a limited region of the dACC (Fig. 1c,d), aiming mainly for portions of Brodmann area 24 (by matching anatomical markers from individual MRI with atlas and previous publications). Accordingly, there was no apparent relationship between anterior-posterior position and the behavioral effect ( $p>0.1$, Pearson correlation coefficient), but there were patches of effect magnitude. Namely, returning to the same location every few days usually obtained a similar magnitude of attenuation of spontaneous recovery $\left(p<0.05, \chi^{2}\right.$ test for nonhomogeneity). Moreover, there was a significant correlation between the depth of the electrode (i.e., closer to the corpuscallosum) and the magnitude of the behavioral effect $(r=0.55$, $p=0.01$, Pearson correlation coefficient), possibly due to more dense projections to the amygdala from these regions (Stefanacci and Amaral, 2002; Ghashghaei et al., 2007).

\section{Low-frequency stimulation is effective for both visual and} auditory CS, and generalizes within but not across modalities We tested whether the stimulation effect was specific to the CS+, generalizes to the CS - of the same modality, or even generalizes to the CS - of the other modality (auditory/visual). To do so, we normalized all behavioral responses to the end of the extinction training for the $\mathrm{CS}+$. This revealed that there were comparable responses and little learning for the two $\mathrm{CS}-$ (Fig. $3 a$, left three bars; $p=0.01, F=4.65$, ANOVA, $p<0.02$ comparing $\mathrm{CS}+$ to either $\mathrm{CS}-$, and $p>0.1$ comparing the two CS - , post hoc tests). Interestingly, the spontaneous recovery generalized to all CS - in a comparable manner (Fig. $3 a$, middle-right bars; Norrholm et al., 2008). Importantly, LFS abolished this generalization for the CS - of the same modality (Fig. $3 a$, middle-right vs right three bars, $p<0.01$ for stimulation and CS - type main effect and interaction, $F=4.94 / 6.2 / 5.83$, two-way ANOVA), but not for the $\mathrm{CS}-$ of the other modality (Fig. $3 a$, right three bars, $p>0.1$, post hoc test comparing stimulation to behavior only). The observation that the response generalized to the CS- of the other modality despite LFS suggests that the stimulation did not abolish all previous immediate learning in an independent manner of the extinction training.

We conclude that discrimination was achieved during acquisition, the memory generalized broadly overnight, but LFS abolished the spontaneous recovery specifically within modality. Finally, the LFS effect was evident when using either modality as the CS + (Fig. 3b, significant effect of stimulation ${ }^{\star}$ learning phase interaction: $F_{(1,86)}=4.0719, p<0.05$ with LSD post hoc revealing significant spontaneous recovery effect only for nonstimulation days only $p<0.0001$ and no effect in stimulation days $p>0.5$.

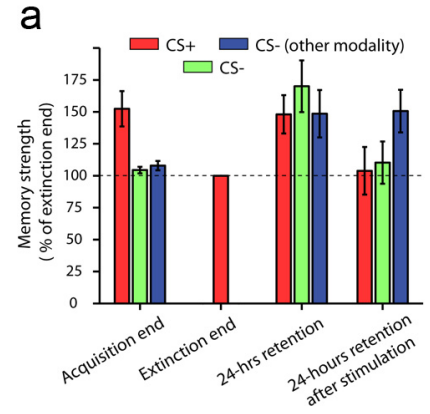

b

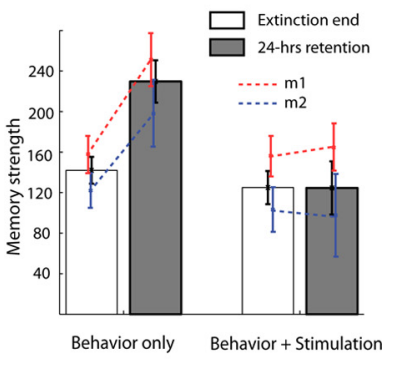

Figure 3. Stimulation induces a within-modality effect, and can be used for either auditory or visual CS. $\boldsymbol{a}$, Conditioned responses (CRs) were normalized to the end of extinction for the $\mathrm{CS}+$, revealing that the acquisition was selective (three leftmost bars), because the responses were comparable between the two $\mathrm{CS}$ - and the end of extinction of the $\mathrm{CS}+$, but also showing that spontaneous recovery $24 \mathrm{~h}$ later generalized broadly and was comparable across all three CS (middle-right bars). The stimulation effect was specific to the modality of the CS+, attenuating spontaneous recovery to the $C S$ - of the same modality, but not to that of the other modality (rightmost bars). See Results, Low-frequency stimulation is effective for both visual and auditory CS, and generalizes within but not across modalities section, for statistics. $\boldsymbol{b}$, Stimulation was able to attenuate recovery when either auditory or visual stimuli were used as the $C S+$. Each dot is a session. The effect was mildly and significantly stronger when auditory stimuli were used as the CS + . Mean \pm SEM is shown next to the dots.

nonsignificant modality main effect $F_{(1,86)}=2.133, p=0.147$ or modality ${ }^{\star}$ stimulation ${ }^{\star}$ learning phase interaction $F_{(1,86)}=1.198$, $p>0.2$ ), albeit it was stronger for the auditory CS (significant modality ${ }^{*}$ stimulation interaction: $F_{(1,86)}=6.035, p<0.05$ with LSD post hoc revealing a reduction in behavior after stimulation in auditory condition compared with all other groups, all $p<$ $0.05)$, potentially due to enhanced access of audition to the amygdala and dACC.

\section{Low-frequency stimulation reduces single-unit and LFP excitability in the dACC}

Our hypothesis, based on previous studies, was that LFS could reduce responsiveness in the dACC. We therefore examined the simultaneously recorded single-units and LFPs. First, we observed a reduction in the typical two-phase evoked field potential (Herry and Garcia, 2002) as a function of stimulation pulses (Fig. $4 a)$. This was reflected in a reduction of peak size, trough size, and the difference between peak and trough as stimulation progressed (Fig. 4b, three different repeated-measure ANOVA of 
a

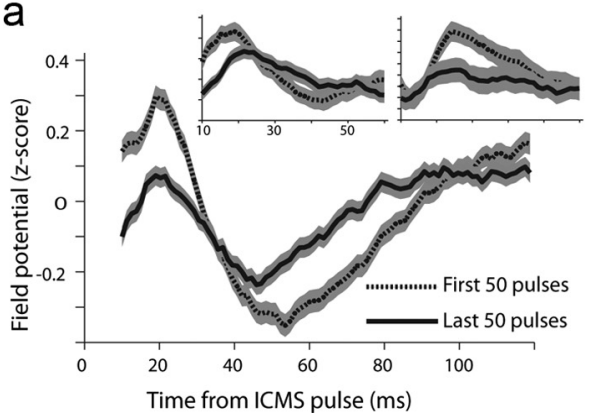

b

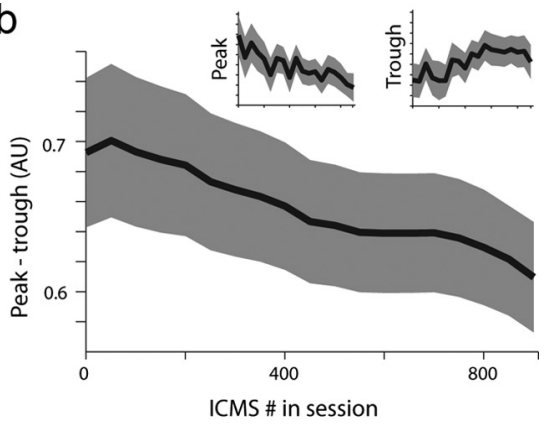

Figure 4. LFS-induced regional reduction in LFP responsiveness. $\boldsymbol{a}$, Evoked responses in local field potentials $(1-200 \mathrm{~Hz})$ averaged over the first 50 pulses and the last 50 pulses, and averaged over all sessions (mean \pm SEM). Insets show data from two representative sessions ( $p<0.01$ for both, ANOVA). $\boldsymbol{b}$, Individual field potentials were $z$-scored to compare across sessions and electrodes. We extracted the peak (maximum) minus the trough (minimum) of the signal, revealing a gradual reduction along with the number of pulses in the session. Insets show peak alone and the trough alone. Data are divided into 19 bins of 50 pulses each and averaged over sessions (mean \pm SEM, $p<0.01$ for all, ANOVA).
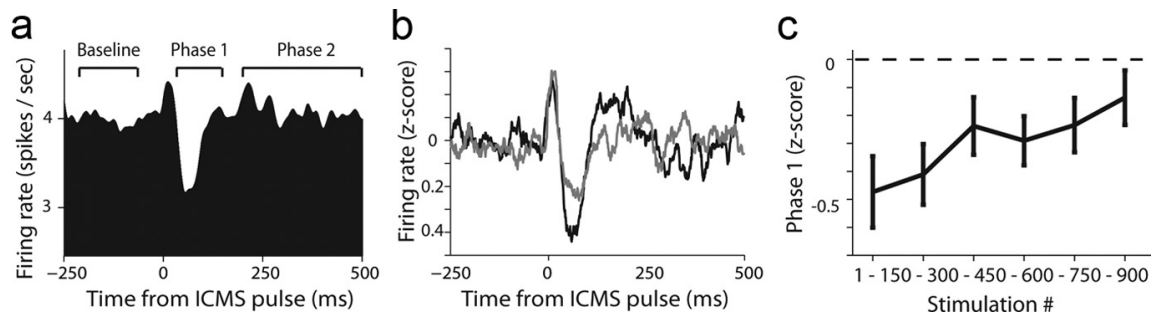

Figure 5. LFS-induced a typical single-unit response that was reduced as a function of stimulation pulses. $\boldsymbol{a}$, Average of raw PSTHs around pulses from all recorded neurons (a similar figure is obtained if $z$-scoring responses first). We define phase 1 as the average firing rate (FR) in $+20 \mathrm{~ms}$ to $+220 \mathrm{~ms}$; and phase 2 as $250 \mathrm{~ms}$ to $500 \mathrm{~ms}$. $\boldsymbol{b}$, PSTH from the first 150 pulses (black) and the last 150 pulses (gray), showing a reduction in both phase 1 and 2 as a result of LFS. The PSTH is averaged over all neurons (as in $\boldsymbol{a}$ ) after z-scoring each neuron for its prepulse baseline. c, Firing rate in phase 1 as a function of stimulation pulses, averaged over all sessions and neurons. The reduction in firing rate became smaller gradually, indicating that the stimulating pulse was less effective.

time-bin were conducted, differing in the dependent variable, all revealed significant effect of stimulation-bin: peak size $\left(F_{(1,18)}=\right.$ $2.742, p<0.001)$, trough size $\left(F_{(1,18)}=2.604, p<0.001\right)$, peak minus trough $\left(F_{(1,18)}=5.4486, p<0.001\right)$; with significant post hoc comparisons between first and last time-bins all $p<0.05$ ).

Single-unit activity showed a typical behavior, where neurons had an initial narrow burst of spikes, then an inhibition phase that lasts $\sim 200 \mathrm{~ms}$, followed by a mild slow compensatory increase in firing rate (Fig. 5a). This 2-phasic response (excluding the initial narrow burst) likely reflects cortical hyperpolarization and depolarization (Seidemann et al., 2002), with the hyperpolarization stage being most prominent and evident in most neurons. We therefore used this phase as a measure for the evoked response, revealing that it indeed gradually decayed to baseline as a function of pulses in session (Fig. $5 b ; p<0.01, F=3.2$, ANOVA; single-cell examples are shown in Fig. 6).

We then tested CS-related responses during extinction and compared neurons recorded during behavior-only sessions to those recorded during LFS sessions. In both types of sessions, neurons showed a gradual reduction in CS-evoked activity (Fig. $7 a, b ; p<0.01$ for both, $F=26.9 / 27.9$, ANOVAs), but neurons in LFS sessions had reduced response all over (in Fig. $7 a, b$; compare $y$-axes). When normalizing the activity of each neuron in each trial to the pre-CS baseline, a similar dynamics was observed (Fig. $7 c$ ). Initial examination of raw responses revealed, as might be expected from the lower firing rates, that variability was also reduced during LFS sessions (Fig. $7 d$ ). To test the trial-to-trial

variability directly, we calculated the SD for each neuron in the first 7 trials and the last 8 trials during extinction. We found that for CS-evoked activity, the variability was decreased in the later part of the extinction, in a similar manner in behavioronly and LFS sessions (Fig. 7e). This can be expected from the reduced firing-rates during LFS and as extinction progresses (Fig. $7 a-c$ ). Interestingly however, we found that although baseline (pre-CS) variability was unchanged during behavior-only days, it was significantly reduced in LFS days as extinction progressed (Fig. $7 f ; p<0.02$, interaction term, $F=5.4$, 2-way ANOVA and $p<$ 0.05 post hoc tests comparing early vs late in LFS sessions and comparing session type in late trials). This might suggest that LFS reduced responsiveness and also decreased baseline variability, allowing a more reliable detection of CS appearance, and hence a more effective extinction process. Because the effect was observed in the last 8 trials of the extinction, after behavior has reached a plateau, it might contribute to later processing of the extinction memory and its competition with the original fear-memory.

\section{Reduction in evoked response predicts overnight reduction in spontaneous recovery}

To test directly the relationship between the physiological effect of LFS and the behavioral effect, we calculated a neural response index for each neuron using the reduction in firing rate in phase 1 (see Fig. $5 a$ ), or for the slow-compensatory increase (phase 2). We found that both phases correlated with the strength of the behavioral effect (measured as the behavior during retention minus that during end of extinction) on a daily basis. Namely, the less hyperpolarization (less reduction in spikes), the less spontaneous recovery there was $24 \mathrm{~h}$ later; and complementary: the less spikes in phase 2 , the less spontaneous recovery. In sum, a smaller evoked response to a pulse in the dACC is linked to less spontaneous recovery $24 \mathrm{~h}$ later (Fig. $8 a, b ; n=61$ neurons recorded in 18 LFS sessions). This, together with the fact that we were able to reduce responsiveness by our LFS protocol (previous subsection), strongly suggests we were able to induce less spontaneous recovery with our stimulation.

\section{Discussion}

The present study tested the effects of LFS $(1 \mathrm{~Hz})$ in the primate dACC when combined with extinction learning of previously acquired aversive associations. Our findings show that LFS significantly attenuated spontaneous recovery when measured after $24-72 \mathrm{~h}$, and this was so when using either visual or auditory modality as the CS+ (in different days). By simultaneously recording neural activity, we show that the LFS resulted in reduction of neuronal excitability in the dACC as stimulation progressed. This was evident in the typical phases of evoked responses of local-field-potentials (LFP), and in firing-rates of single-units. Importantly, the reduction in memory retention 
that was measured $24 \mathrm{~h}$ after stimulation was correlated with the reduced excitability in the dACC during stimulation. Finally, we found the behavioral effect to generalize within modality, but not generalize (be specific) across modalities. We discuss these findings below.

Low-frequency stimulation is a protocol that was shown to induce long-term depression (LTD), a sustained decrease in synaptic strength (Malenka, 1994). Because synaptic plasticity is thought to play a major role in learning and memory, artificial induction received much attention. Synaptic changes were demonstrated in vitro by measuring changes in field potentials (FP), and LTD was demonstrated as a reduction in the size of the typical FP complex which appears after stimulation (Dudek and Bear, 1992; Kirkwood et al., 1993). In behaving animals, synaptic plasticity is traditionally demonstrated by comparing evoked responses of local-field-potentials (LFP) along different time points in the stimulation protocol (Herry and Garcia, 2002; Maroun, 2006; Hugues and Garcia, 2007). In the present study, stimulation was applied every second for a total of $15 \mathrm{~min}$ (900 pulses overall) and evoked responses were measured around each stimulation event. A prototypic LFP response and a prototypic singleunits' response (Seidemann et al., 2002) were identified and we compared how they change as stimulation progressed. For LFP, we identified that the two typical phases (peak and trough) decay; and for firing rate of single-units, we observed that the typical 3 -phasic response also decays. Although it is hard to obtain direct evidence for LTD in extracellular recordings in behaving animals (and primates especially), we suggest that the reduction in excitability that is evident in all our measures and relies on the traditional ways to identify synaptic strength, shows that we were able to reduce regional responsiveness in the dACC by using LFS. The stimulating and recording electrodes were positioned $1-4 \mathrm{~mm}$ apart and we did not detect any trend of effect-magnitude as a function of distance between the two (data not shown, $p>0.1$, ANOVA), further suggesting that it relies on changes in horizontal connectivity within the dACC, and comparable to previous studies of cortical stimulation (Seidemann et al., 2002).

The results suggest a major role for the primate dACC in maintenance and expression of aversive memories. In principle, attenuation of spontaneous recovery could be achieved by affecting the strength of extinction (Milad and Quirk, 2002; Sierra-Mercado et al., 2011), or of the original memory. In the present case, it seems the latter is more likely to be the mechanism; namely, it seems more likely that the effect of LFS was on the original CS-US contingency. First, because we stimulated in the dACC, an area related to fear-expression (Dunsmoor et al., 2007; Milad et al., 2007), and depressed it. Second, we did not observe any significant reduction of memory expression or of learning-rate during extinction (Sierra-Mercado et al., 2011), but only following overnight retention. Finally, spontaneous recovery after extinction is commonly accepted as an indicator for the original memory that
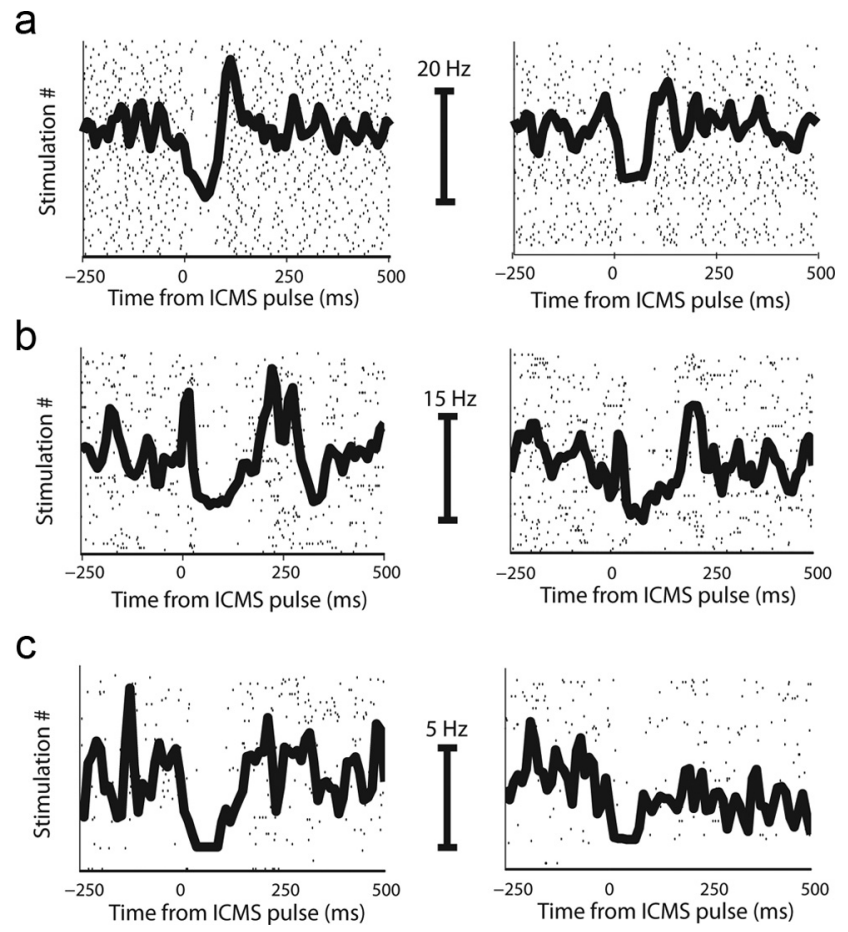

Figure 6. Single-unit examples of reduction in evoked response. $\boldsymbol{a}-\boldsymbol{c}$, Three single-cell examples of a reduction in evoked response to the stimulation. Each row is one neuron. Left column shows the response to the first 100 pulses, and right column shows the response to the last 100 pulses in the session. Shown are rasters overlaid with the peristimulus-time histogram (PSTH). Calibrating bars indicate the firing rate in each pair of PSTHs.
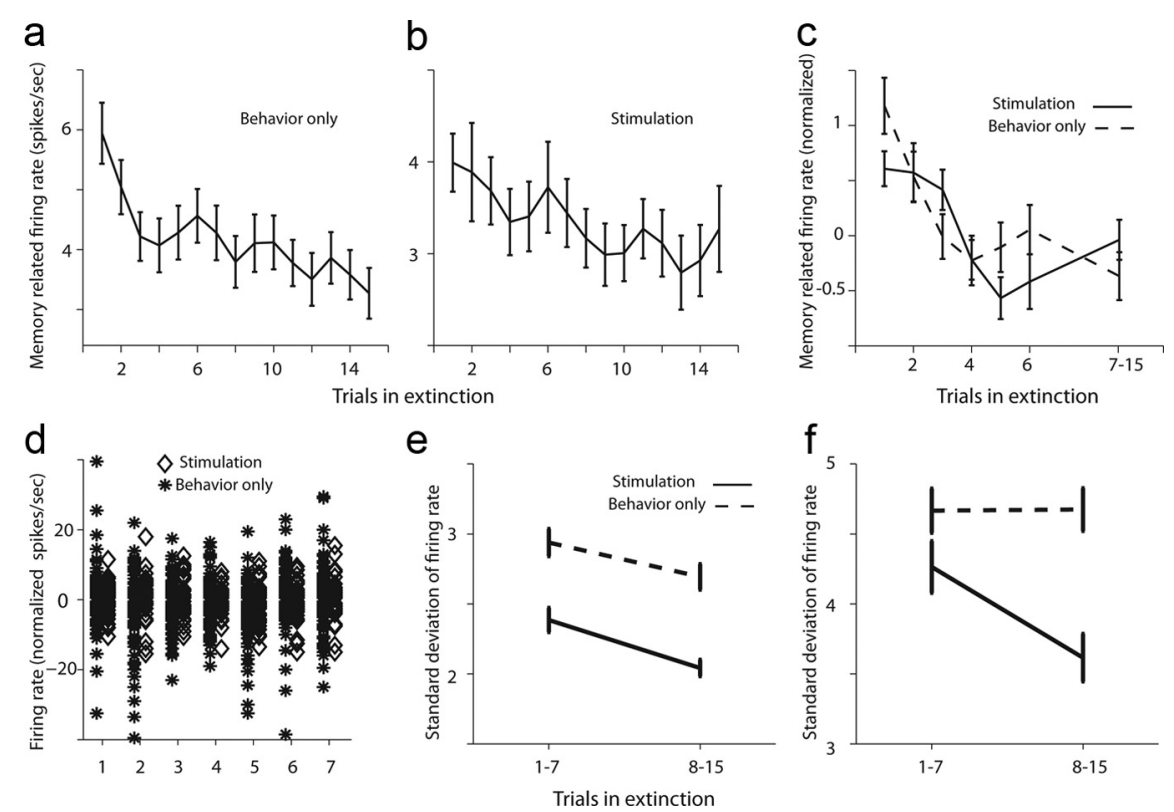

Figure 7. Neurons reduce their CS related activity and trial-to-trial variability during extinction. $\boldsymbol{a}$, Firing rate in the 2 s post-CS (similar to the time-frame used for behavior), averaged over all neurons in behavior-only sessions ( $n=207$ neurons). There was a gradual significant reduction. $\boldsymbol{b}$, Same as in $\boldsymbol{a}$ for LFS sessions ( $n=74$ neurons). There was a gradual significant reduction. In addition, notice that the overall firing rate is reduced compared with $\boldsymbol{a}$ ( $y$-axes). $c$, Normalized firing rate (post-CS minus pre-CS) for both behavior-only (dashed) and LFS (solid) sessions. $\boldsymbol{d}$, Normalized rates from all neurons in behavior-only (asterisks to the left) and LFS (diamonds to the right) in the first 7 extinction trials. There was overall reduced variability and rates in LFS sessions. $\boldsymbol{e}$, The SD of post-CS firing rates over trials 1-7 and trials 8-15, for behavior-only (dashed) and LFS (solid) sessions. Trial-to-trial variability was reduced with extinction training in both. $\boldsymbol{f}$, The same as in $\boldsymbol{e}$ but for baseline (pre-CS) activity. Variability was reduced with extinction only for LFS sessions, but not for behavior-only sessions. 
a

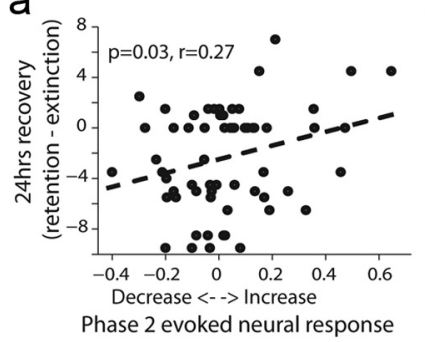

b

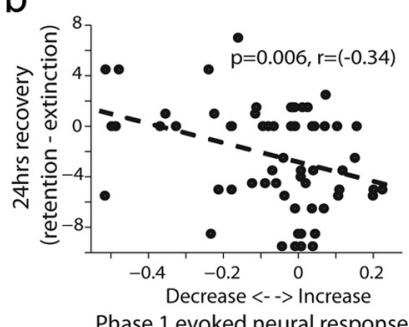

Figure 8. The magnitude of single-unit response in a particular session is correlated with the behavioral reduction in spontaneous recovery $24 \mathrm{~h}$ later. $\boldsymbol{a}$, Correlation between firing rate (FR) in phase 2 and the behavioral effect (spontaneous recovery). The evoked response index is defined as (FR_Phase2-FR_Baseline)/(FR_Phase2 + FR_Baseline). Below zero means a reduction in FR and $>0$ an increase. The behavioral response is defined as the $C R$ in the retention minus the $C R$ at the end of previous day extinction. The significant correlation shows that the less spikes there were in Phase 2 , the less spontaneous was recovery there. Shown are $n=61$ neurons recorded in 18 LFS sessions. All neurons with firing rate of $>0.5$ spikes $/ \mathrm{s}$ in phase 1 and 2 took part in the analysis. $\boldsymbol{b}$, Same as in $\boldsymbol{a}$, but when taking the FR from Phase 1 . Hence, the less reduction in FR, the less spontaneous was recovery. Notice that the reduction in FR in this Phase is an evidence to the effectiveness of the pulse, and most likely to the regional synaptic efficacy.

competes with the new inhibitory one (Robbins, 1990; Bouton, 1993; Monfils et al., 2009), and here, we chose to use extinction that immediately follows acquisition, because several studies have observed significant recovery in this protocol (Rescorla, 2004; Maren and Chang, 2006; Alvarez et al., 2007; Schiller et al., 2008; Woods and Bouton, 2008). However, it is important to note that different mechanisms could underlie immediate versus delayed extinction (Myers et al., 2006), and behavior varies across species and paradigms (Norrholm et al., 2008). In addition, we cannot conclude that spontaneous recovery would not still occur after longer time-frames, i.e., more than the $72 \mathrm{~h}$ here.

In contrast to studies that abolish the spontaneous activity of the PL by HFS (Vidal-Gonzalez et al., 2006), or Muscimol (Blum et al., 2006; Corcoran and Quirk, 2007; Laurent and Westbrook, 2009), the local effect of LFS is brief and minor (Hashimoto et al., 2003; Maurice et al., 2003; Bar-Gad et al., 2004); yet LFS has a long-term accumulating effect on the region's responsiveness, as shown here (Mockett et al., 2002). Further, stimulation was not coupled with presentation and therefore did not affect behavioral expression and CS-related responses. However, there was a global gradual effect, on both responsiveness and baseline trial-to-trial variability that most likely influenced later processing. It could suggest a role for the dACC in consolidation/recall of the original memory, and harming it by LFS therefore results in less spontaneous recovery. In addition, because the dACC is implicated in uncertainty and error-detection (Rushworth and Behrens, 2008; Bryden et al., 2011; Hayden et al., 2011), the gradual reduction in responsiveness could further harm processing of the original memory (that now involves uncertainty and predictionerror during extinction). A most likely possibility is that because extinction is learned and expressed by a different pathway, that of the amygdala-vmPFC (or the rodent IL; Milad and Quirk, 2002; Phelps et al., 2004; Sierra-Mercado et al., 2011), it had an "easier" job of competing with the original memory that gradually received less support from dACC inputs due to the LFS.

Modification of the original memory can occur in a similar way to what happens during reconsolidation paradigms (Nader

and Hardt, 2009): the memory that is currently retrieved and active (because of CS presentation during extinction) is being modified, even without the use of a reconsolidation paradigm per se (Dudai, 2009). Further supporting this notion, the effect of stimulation was specific to the modality being presented during extinction, but did not generalize to the other modality. The initial (without LFS) broad generalization after extinction to all CS presented during the acquisition is an interesting phenomena in itself and was previously observed in fear learning, and even shown to be enhanced with protocols of immediate extinction (Norrholm et al., 2008). This broad generalization could be due to the habituation session in which all stimuli were presented and primed, as suggested for perceptual learning (Sagi, 2011); however, a reverse effect of pre-exposure was found for feargeneralization (Vervliet et al., 2010). Generalization of fear is an important aspect of post-traumatic stress disorder (PTSD) and anxiety-disorders in general (Lissek et al., 2009; Jovanovic et al., 2010; Dunsmoor et al., 2011; Resnik et al., 2011), but less is known about generalization of the extinction process as an indicator for therapy (Vervliet et al., 2006). Although the exact extent of this generalization is a complex issue in real-life psychiatric disorders, a balance of specificity and some generalization, as described here, is a necessary requirement from any successful treatment.

There are two interpretational limitations of our study. The first relates to the lack of a stimulation-only control group. One could argue that the effect observed is due to the stimulation independent of the extinction training. However, we think that the aforementioned finding, of generalization of the aversive memory to the other modality that was not abolished by the LFS, suggests that this is not the case. In other words, if the LFS would retroactively inhibit all memories independent of which was active at the time (due to extinction), we would not observe any aversive-memory in the following day as we did for the other modality.

The other limitation relates to the frequency of stimulation. We used only low-frequency $(1 \mathrm{~Hz})$, based on previous findings that it reduces regional and synaptic excitability, and we demonstrated with electrophysiology that it did so. Another major goal of using LFS in the primate was to show that a relatively subtle protocol, in a relatively accessible cortex, can induce the required behavioral effect. Although we cannot conclude whether higherfrequencies of stimulation would not obtain the same behavioral results, we think there is large benefit in showing that this protocol works in the primate brain.

Whereas deep brain stimulation (DBS) is becoming more common as a possible treatment for several psychiatric disorders (Wichmann and Delong, 2006; Gubellini et al., 2009); to the best of our knowledge, its application for PTSD remains elusive. Standard DBS usually replaces regional ablation by using highfrequency stimulation and simulating clinical outcomes of a lesion by suppression of spontaneous activity (McIntyre et al., 2004). Here we offer a new approach by taking advantage of more subtle stimulation schedules (LFS, low-amplitude) to induce synaptic plasticity. Moreover, targeting dACC, rather than deeper subcallosal regions associated with extinction (vmPFC; Phelps et al., 2004; Sierra-Mercado et al., 2011), could be less invasive and amenable for standard trans-magnetic-stimulation (TMS) that can also reduce excitability with low-frequency protocols (Pascual-Leone et al., 1998). All in all, the aim would be to combine behavioral invaluable therapy with stimulation, and achieve less maintenance and/or spontaneous recovery of aversive memories in severe cases. 


\section{References}

Alvarez RP, Johnson L, Grillon C (2007) Contextual-specificity of shortdelay extinction in humans: renewal of fear-potentiated startle in a virtual environment. Learn Mem 14:247-253.

Amano T, Unal CT, Paré D (2010) Synaptic correlates of fear extinction in the amygdala. Nat Neurosci 13:489-494.

Bar-Gad I, Elias S, Vaadia E, Bergman H (2004) Complex locking rather than complete cessation of neuronal activity in the globus pallidus of a 1-methyl-4-phenyl-1,2,3,6-tetrahydropyridine-treated primate in response to pallidal microstimulation. J Neurosci 24:7410-7419.

Blum S, Hebert AE, Dash PK (2006) A role for the prefrontal cortex in recall of recent and remote memories. Neuroreport 17:341-344.

Bouton ME (1993) Context, time, and memory retrieval in the interference paradigms of Pavlovian learning. Psychol Bull 114:80-99.

Bouton ME (2006) Learning and behavior: a contemporary synthesis. Sunderland, MA: Sinauer.

Bremner JD, Vermetten E, Schmahl C, Vaccarino V, Vythilingam M, Afzal N, Grillon C, Charney DS (2005) Positron emission tomographic imaging of neural correlates of a fear acquisition and extinction paradigm in women with childhood sexual-abuse-related post-traumatic stress disorder. Psychol Med 35:791-806.

Bryden DW, Johnson EE, Tobia SC, Kashtelyan V, Roesch MR (2011) Attention for learning signals in anterior cingulate cortex. J Neurosci 31:18266-18274.

Corcoran KA, Quirk GJ (2007) Activity in prelimbic cortex is necessary for the expression of learned, but not innate, fears. J Neurosci 27:840-844.

Delgado MR, Olsson A, Phelps EA (2006) Extending animal models of fear conditioning to humans. Biol Psychol 73:39-48.

Dudai Y (2009) Predicting not to predict too much: how the cellular machinery of memory anticipates the uncertain future. Philos Trans R Soc Lond B Biol Sci 364:1255-1262.

Dudek SM, Bear MF (1992) Homosynaptic long-term depression in area CA1 of hippocampus and effects of N-methyl-D-aspartate receptor blockade. Proc Natl Acad Sci U S A 89:4363-4367.

Dunsmoor JE, Bandettini PA, Knight DC (2007) Impact of continuous versus intermittent CS-UCS pairing on human brain activation during Pavlovian fear conditioning. Behav Neurosci 121:635-642.

Dunsmoor JE, Prince SE, Murty VP, Kragel PA, LaBar KS (2011) Neurobehavioral mechanisms of human fear generalization. Neuroimage 55:1878-1888.

Duvarci S, Popa D, Paré D (2011) Central amygdala activity during fear conditioning. J Neurosci 31:289-294.

Foa EB (2006) Psychosocial therapy for posttraumatic stress disorder. J Clin Psychiatry 67 [Suppl 2]:40-45.

Ghashghaei HT, Hilgetag CC, Barbas H (2007) Sequence of information processing for emotions based on the anatomic dialogue between prefrontal cortex and amygdala. Neuroimage 34:905-923.

Goosens KA, Maren S (2004) NMDA receptors are essential for the acquisition, but not expression, of conditional fear and associative spike firing in the lateral amygdala. Eur J Neurosci 20:537-548.

Gubellini P, Salin P, Kerkerian-Le Goff L, Baunez C (2009) Deep brain stimulation in neurological diseases and experimental models: from molecule to complex behavior. Progress in neurobiology 89:79-123.

Hashimoto T, Elder CM, Okun MS, Patrick SK, Vitek JL (2003) Stimulation of the subthalamic nucleus changes the firing pattern of pallidal neurons. J Neurosci 23:1916-1923.

Hayden BY, Heilbronner SR, Pearson JM, Platt ML (2011) Surprise signals in anterior cingulate cortex: neuronal encoding of unsigned reward prediction errors driving adjustment in behavior. J Neurosci 31:4178-4187.

Herry C, Garcia R (2002) Prefrontal cortex long-term potentiation, but not long-term depression, is associated with the maintenance of extinction of learned fear in mice. J Neurosci 22:577-583.

Herry C, Ciocchi S, Senn V, Demmou L, Müller C, Lüthi A (2008) Switching on and off fear by distinct neuronal circuits. Nature 454:600-606.

Hugues S, Garcia R (2007) Reorganization of learning-associated prefrontal synaptic plasticity between the recall of recent and remote fear extinction memory. Learning and Memory 14:520-524.

Jovanovic T, Norrholm SD, Blanding NQ, Davis M, Duncan E, Bradley B, Ressler KJ (2010) Impaired fear inhibition is a biomarker of PTSD but not depression. Depress Anxiety 27:244-251.

Kim J, Lee S, Park K, Hong I, Song B, Son G, Park H, Kim WR, Park E, Choe HK, Kim H, Lee C, Sun W, Kim K, Shin KS, Choi S (2007) Amygdala depotentiation and fear extinction. Proc Natl Acad Sci U S A 104: 20955-20960.

Kirkwood A, Dudek SM, Gold JT, Aizenman CD, Bear MF (1993) Common forms of synaptic plasticity in the hippocampus and neocortex in vitro. Science 260:1518-1521.

Laurent V, Westbrook RF (2009) Inactivation of the infralimbic but not the prelimbic cortex impairs consolidation and retrieval of fear extinction. Learn Mem 16:520-529.

LeDoux JE (2000) Emotion circuits in the brain. Annu Rev Neurosci 23:155-184.

Liberzon I, Taylor SF, Amdur R, Jung TD, Chamberlain KR, Minoshima S, Koeppe RA, Fig LM (1999) Brain activation in PTSD in response to trauma-related stimuli. Biol Psychiatry 45:817-826.

Likhtik E, Pelletier JG, Paz R, Paré D (2005) Prefrontal control of the amygdala. J Neurosci 25:7429-7437.

Lissek S, Rabin S, Heller RE, Lukenbaugh D, Geraci M, Pine DS, Grillon C (2010) Overgeneralization of conditioned fear as a pathogenic marker of panic disorder. Am J Psychiatry 167:47-55.

Malenka RC (1994) Synaptic plasticity in the hippocampus: LTP and LTD. Cell 78:535-538.

Maren S, Chang CH (2006) Recent fear is resistant to extinction. Proc Natl Acad Sci U S A 103:18020-18025.

Maren S, Quirk GJ (2004) Neuronal signalling of fear memory. Nat Rev Neurosci 5:844-852.

Maroun M (2006) Stress reverses plasticity in the pathway projecting from the ventromedial prefrontal cortex to the basolateral amygdala. Eur J Neurosci 24:2917-2922.

Martin RF, Bowden DM (2000) Primate brain maps: structure of the macaque brain. Amsterdam: Elsevier Science.

Maurice N, Thierry AM, Glowinski J, Deniau JM (2003) Spontaneous and evoked activity of substantia nigra pars reticulata neurons during highfrequency stimulation of the subthalamic nucleus. J Neurosci 23:9929-9936.

McDonald AJ (1998) Cortical pathways to the mammalian amygdala. Prog Neurobiol 55:257-332.

McIntyre CC, Savasta M, Kerkerian-Le Goff L, Vitek JL (2004) Uncovering the mechanism(s) of action of deep brain stimulation: activation, inhibition, or both. Clin Neurophysiol 115:1239-1248.

Milad MR, Quirk GJ (2002) Neurons in medial prefrontal cortex signal memory for fear extinction. Nature 420:70-74.

Milad MR, Rauch SL, Pitman RK, Quirk GJ (2006) Fear extinction in rats: implications for human brain imaging and anxiety disorders. Biol Psychol 73:61-71.

Milad MR, Quirk GJ, Pitman RK, Orr SP, Fischl B, Rauch SL (2007) A role for the human dorsal anterior cingulate cortex in fear expression. Biol Psychiatry 62:1191-1194.

Milad MR, Pitman RK, Ellis CB, Gold AL, Shin LM, Lasko NB, Zeidan MA, Handwerger K, Orr SP, Rauch SL (2009) Neurobiological basis of failure to recall extinction memory in posttraumatic stress disorder. Biol Psychiatry 66:1075-1082.

Mockett B, Coussens C, Abraham WC (2002) NMDA receptor-mediated metaplasticity during the induction of long-term depression by lowfrequency stimulation. Eur J Neurosci 15:1819-1826.

Monfils MH, Cowansage KK, Klann E, LeDoux JE (2009) Extinctionreconsolidation boundaries: key to persistent attenuation of fear memories. Science 324:951-955.

Myers KM, Davis M (2007) Mechanisms of fear extinction. Mol Psychiatry 12:120-150.

Myers KM, Ressler KJ, Davis M (2006) Different mechanisms of fear extinction dependent on length of time since fear acquisition. Learn Mem 13:216-223.

Nader K, Hardt O (2009) A single standard for memory: the case for reconsolidation. Nat Rev Neurosci 10:224-234.

Norrholm SD, Vervliet B, Jovanovic T, Boshoven W, Myers KM, Davis M, Rothbaum B, Duncan EJ (2008) Timing of extinction relative to acquisition: A parametric analysis of fear extinction in humans. Behav Neurosci 122:1016-1030.

Pannu Hayes J, Labar KS, Petty CM, McCarthy G, Morey RA (2009) Alterations in the neural circuitry for emotion and attention associated with posttraumatic stress symptomatology. Psychiatry Res 172:7-15.

Pape HC, Pare D (2010) Plastic synaptic networks of the amygdala for the 
'acquisition, expression, and extinction of conditioned fear. Physiol Rev 90:419-463.

Pascual-Leone A, Tormos JM, Keenan J, Tarazona F, Cañete C, Catalá MD (1998) Study and modulation of human cortical excitability with transcranial magnetic stimulation. J Clin Neurophysiol 15:333-343.

Paton JJ, Belova MA, Morrison SE, Salzman CD (2006) The primate amygdala represents the positive and negative value of visual stimuli during learning. Nature 439:865-870,

Pavlov I (1927) Conditioned reflexes. London: Oxford UP.

Phelps EA, Delgado MR, Nearing KI, LeDoux JE (2004) Extinction learning in humans: role of the amygdala and vmPFC. Neuron 43:897-905.

Quirk GJ, Paré D, Richardson R, Herry C, Monfils MH, Schiller D, Vicentic A (2010) Erasing fear memories with extinction training. J Neurosci 30:14993-14997.

Rescorla RA (2004) Spontaneous recovery varies inversely with the training-extinction interval. Learn Behav 32:401-408.

Resnik J, Sobel N, Paz R (2011) Auditory aversive learning increases discrimination thresholds. Nat Neurosci 14:791-796.

Robbins SJ (1990) Mechanisms underlying spontaneous recovery in autoshaping. J Exp Psychol: Animal Behav Proc 16:235-249.

Rushworth MF, Behrens TE (2008) Choice, uncertainty and value in prefrontal and cingulate cortex. Nat Neurosci 11:389-397.

Sagi D (2011) Perceptual learning in vision research. Vision Res 51:1552-1566.

Saleem K, Logothetis N (2007) A combined MRI and histology atlas of the rhesus monkey brain. London: Elsevier.

Schiller D, Cain CK, Curley NG, Schwartz JS, Stern SA, Ledoux JE, Phelps EA (2008) Evidence for recovery of fear following immediate extinction in rats and humans. Learn Mem 15:394-402.

Seidemann E, Arieli A, Grinvald A, Slovin H (2002) Dynamics of depolarization and hyperpolarization in the frontal cortex and saccade goal. Science 295:862-865.

Shin LM, Bush G, Milad MR, Lasko NB, Brohawn KH, Hughes KC, Macklin
ML, Gold AL, Karpf RD, Orr SP, Rauch SL, Pitman RK (2011) Exaggerated activation of dorsal anterior cingulate cortex during cognitive interference: a monozygotic twin study of posttraumatic stress disorder. Am J Psychiatry 168:979-985.

Sierra-Mercado D, Padilla-Coreano N, Quirk GJ (2011) Dissociable roles of prelimbic and infralimbic cortices, ventral hippocampus, and basolateral amygdala in the expression and extinction of conditioned fear. Neuropsychopharmacology 36:529-538.

Stefanacci L, Amaral DG (2002) Some observations on cortical inputs to the macaque monkey amygdala: an anterograde tracing study. J Comp Neurol 451:301-323.

Straube T, Glauer M, Dilger S, Mentzel HJ, Miltner WH (2006) Effects of cognitive-behavioral therapy on brain activation in specific phobia. Neuroimage 29:125-135.

Vervliet B, Vansteenwegen D, Eelen P (2006) Generalization gradients for acquisition and extinction in human contingency learning. Exp Psychol 53:132-142.

Vervliet B, Kindt M, Vansteenwegen D, Hermans D (2010) Fear generalization in humans: impact of prior non-fearful experiences. Behav Res Ther 48:1078-1084.

Vidal-Gonzalez I, Vidal-Gonzalez B, Rauch SL, Quirk GJ (2006) Microstimulation reveals opposing influences of prelimbic and infralimbic cortex on the expression of conditioned fear. Learn Mem 13:728-733.

Wang SJ, Gean PW (1999) Long-term depression of excitatory synaptic transmission in the rat amygdala. J Neurosci 19:10656-10663.

Wichmann T, Delong MR (2006) Deep brain stimulation for neurologic and neuropsychiatric disorders. Neuron 52:197-204.

Woods AM, Bouton ME (2008) Immediate extinction causes a less durable loss of performance than delayed extinction following either fear or appetitive conditioning. Learn Mem 15:909-920.

Yehuda R, LeDoux J (2007) Response variation following trauma: a translational neuroscience approach to understanding PTSD. Neuron 56:19-32. 